\title{
Erratum to: Cloning, expression, purification, antiserum preparation and its characteristics of the truncated UL6 protein of herpes simplex virus 1
}

\author{
Meili Li • Wei Cui • Chuncong Mo • \\ Jinlin Wang $\cdot$ Zhiyao Zhao $\cdot$ Mingsheng Cai
}

Published online: 31 August 2014

(C) Springer Science+Business Media Dordrecht 2014

Erratum to: Mol Biol Rep (2014) 41:5997-6002

DOI 10.1007/s11033-014-3477-y

Acknowledgements should be correctly read as:

This work was supported by grants from the National Natural Science Foundation of China (31200120 and 31400150); the Natural Science Foundation of Guangdong
Province (S2013040016596); the Foundation for Distinguished Young Talents in Higher Education of Guangdong, China (2013LYM_0096); the Science and Technology New Star in Zhu Jiang, Guangzhou City (2013J2200018); and the First Batch of Young Core Instructor of Guangzhou Medical University. Dr. Yasushi Kawaguchi is gratefully acknowledged for generous gift of pYEbac102.
The online version of the original article can be found under doi:10.1007/s11033-014-3477-y.

Authors: Meili Li and Wei Cui contributed equally to this work and should each be considered as a first author.

M. Li · W. Cui · C. Mo · J. Wang - Z. Zhao · M. Cai $(\bowtie)$ Department of Pathogenic Biology and Immunology, Guangzhou Medical University, 195 Dongfeng Xi Road, Yuexiu, Guangzhou 510182, Guangdong, People's Republic of China e-mail: mingshengcai@hotmail.com

M. Li · M. Cai

Institute of Immunology, Sino-French Hoffmann Institute,

Guangzhou Medical University, Guangzhou 510182,

People's Republic of China 